\title{
Analysis of Spectral Features of EEG signal in Brain Tumor Condition
}

\author{
V. Salai Selvam ${ }^{1}$, S. Shenbaga Devi ${ }^{2}$ \\ ${ }^{1}$ Sriram Engineering College, Department of Electronics \& Communication Engineering, Chennai - 602 024, India, \\ vsalaiselvam@yahoo.com \\ ${ }^{2}$ College of Engineering, Department of Electronics \& Communication Engineering, Faculty of Information \& \\ Communication, Anna University, Guindy, Chennai - 600 025, s_s_devi@annauniv.edu
}

\begin{abstract}
The scalp electroencephalography (EEG) signal is an important clinical tool for the diagnosis of several brain disorders. The objective of the presented work is to analyze the feasibility of the spectral features extracted from the scalp EEG signals in detecting brain tumors. A set of $\mathbf{1 6}$ candidate features from frequency domain is considered. The significance on the mean values of these features between 100 brain tumor patients and 102 normal subjects is statistically evaluated. Nine of the candidate features significantly discriminate the brain tumor case from the normal one. The results encourage the use of (quantitative) scalp EEG for the diagnosis of brain tumors.
\end{abstract}

Keywords: Brain tumor, electroencephalography, power spectrum, bispectrum, bicoherence, spectral entropy.

\section{INTRODUCTION}

$I^{\mathrm{N}}$ $\mathrm{N}$ SPITE of several advancements in the neuroimaging techniques, there is still a considerable pre-diagnostic symptomatic interval (PSI) (the time interval from the onset of first symptom to the diagnosis) in the diagnosis of brain tumors [1]-[5]. The PSI ranges from few days to over a year in around $25 \%$ of cases. This is due to the following facts: (i) the misleading general symptoms, (ii) the unavailability of neuroimaging facilities, and (iii) the risk and cost involved in the neuroimaging techniques. The firstlevel symptoms such as headache (in around $85 \%$ cases), strange feeling in the head (in around $56 \%$ cases), and nausea/vomiting (in around $43 \%$ cases) are more general and common to several diseases. The neuroimaging facilities are not available in all clinics, especially in those situated in the rural areas of developing countries. The neuroimaging techniques such as the magnetic resonance imaging (MRI) and the computed tomography (CT) do involve a complicated procedure with certain risk of safety hazards, e.g., static electromagnetic field and radiation, and discomfort to the patients, e.g., fear and dizziness, which restricts the physicians from using them willingly [6]. However, it is necessary to reduce the PSI in order to increase the survival rate of the patients [3], [4]. One possible alternative is the scalp electroencephalogram (EEG) [4].

Since the introduction of the term, 'delta waves' and its association with the brain tumors of cerebral hemisphere by Walter [7], many researchers [8]-[14], though the neuroimaging techniques alone can produce confirmative results, have qualitatively investigated the use of scalp EEG for the diagnosis of brain tumors. However, only few researchers [15]-[20] have conducted some quantitative investigations on this issue, such as automated classification or localization using the quantitative (scalp) EEG (qEEG) features with encouraging results.

The recording of scalp EEG is non-invasive, free of risky radiations or drugs and cheaper in terms of equipment cost, computational complexity in producing reliable results, storage requirement and portability [6]. The main difficulties in the EEG recording process are the requirement of the patient's cooperation and the removal of artifacts.

The EEG abnormalities in the presence of a brain tumor include [11], [21] (i) the polymorphic delta activity (PDA) (especially localized, persistent and non-reactive), (ii) the intermittent rhythmic delta activity (IRDA) (especially frontal), (iii) slowing of background rhythm (especially alpha), (iv) complete or incomplete loss of electrical activity in and around the tumor location, (v) disturbance of alpha rhythm, (vi) amplitude asymmetry in the beta rhythm, and (vii) epileptiform discharges. Generally, the presence of a brain tumor causes the background (normal) EEG rhythm to slow down. The EEG abnormalities can be either focal or diffused [4], [10]. The focal abnormalities can further be either ipsilateral or contralateral or bilateral. The abnormalities are dependent on the type, location, rate of growth of the tumor and time.

In this paper, the results of the investigations on the ability of several spectral features in discriminating the multichannel EEG signals of a brain tumor patient from those of a normal subject are presented.

\section{MATERIALS AND METHODS}

Data were collected from the Institute of Neurology, Madras Medical College (MMC), Chennai, Tamil Nadu, India, after getting approval from the Ethical Committee of the Institute. The Department of Neurosurgery of the Institute selected the patients with clinical evidence of brain tumor and the Department of Neurology of the Institute recorded the scalp EEG from them after completing the required formalities.

The method consists of selecting a set of relevant candidate features from the frequency domain followed by the extraction of these features from the EEG records of the normal and brain tumor cases and finally statistically analyzing their ability in discriminating the cases. 


\section{A. Data set}

Nineteen-channel, common reference montage (referenced to linked earlobes) scalp EEG records, in the standard 10-20 electrode system, from 100 brain tumor patients (subjects with clinical evidence of brain tumor) at rest with eyes closed, were obtained at a sampling rate of $256 \mathrm{~Hz}$. Similar records were obtained from 102 normal subjects (subjects without any clinical evidence of brain tumors).

\section{B. Preprocessing}

The EEG records were obtained with minimal artifacts through proper recording conditions laid by the experts from the Department of Neurology of MMC. Certain artifacts such as eye movements, eye rolling and essential tremors, which, generally, fall in the useful bandwidth of cerebral EEG, were removed offline by a recently proposed combination of independent component analysis (ICA) technique and discrete wavelet transform known as the modified wavelet ICA. All EEG records were then bandpass-filtered to $1-40 \mathrm{~Hz}$ as the signals below $1 \mathrm{~Hz}$ and above $40 \mathrm{~Hz}$ in EEG are generally unreliable due to low signal-to-noise ratio [22], [23]. The records were rereferenced to common average reference to approximate a reference-free recording condition [23], to minimize the artifacts [24], [25], to make channel records independent i.e., to make channel records to represent local activities [24], [26], and to provide high reliability over quantitative EEG features [27]. Finally a 10-minute, artifact-free epoch from each EEG record was retained for the analysis.

\section{Selection of features}

A set of 'relevant' features was manually selected based on some heuristic assessment over their relevance and the computational complexity involved in the estimation of the features. The heuristic assessment over the relevance is based on the facts observed by the physicians on the characteristics of the scalp EEG in relevance to the brain tumors such as the gain in low frequency rhythms, the loss in high frequency rhythms, the perturbations in normal background rhythms, the discriminative characteristic waves of EEG that are indicative of brain tumors, e.g., PDA and the changes in the EEG dynamical structure, e.g., the waveform complexity. Since only few literature sources on the quantitative analysis of the issue are available, relatively 'equivalent' literature sources on the EEG and other time series analyses were considered to arrive at some heuristic assessments on the selection of features. The heuristically selected features have been briefly described below.

1) Power Ratio Index (PRI)

The $P R I$ is the ratio of power in one band to that in another. Given a discrete-time signal, $x(n), n=0,1,2, \ldots$, $N-1$ of length, $N$, the PRI is calculated as follows. The power spectrum, $P_{x}(f)$ of the signal, $x(n)$ is estimated using the Welch method of averaged periodograms [28] and then the $P R I$ is defined as

$$
P R I=\sum_{f=f_{11}}^{f_{12}} P_{x}(f) / \sum_{f=f_{j 1}}^{f_{f_{2}}} P_{x}(f)
$$

where $\left[f_{i 1}, f_{i 2}\right]$ and $\left[f_{j 1}, f_{j 2}\right], i \neq j$ are the edges of bands considered. In this study the ratio of power in the delta-theta band $(1-8 \mathrm{~Hz})$ to that in the alpha-beta band $(8-30 \mathrm{~Hz})$ as an indicative of gain (loss) in low (high) frequency power [19] is considered.

\section{2) Relative Intensity Ratio (RIR)}

The $R I R$ is the ratio of power in a band to the total power i.e., a measure of relative power in an EEG band. Thus, the $R I R$ is defined as [29]

$$
R I R_{i}=\sum_{f=f_{11}}^{f_{12}} P_{x}(f) / \sum_{f} P_{x}(f)
$$

where $\left[f_{i 1}, f_{i 2}\right]$ are the edges of bands considered. All the five conventional EEG bands are considered in this study i.e., $[1,4] \mathrm{Hz}$ for the delta $(i=d),[4,8] \mathrm{Hz}$ for theta $(i=t),[8,13]$ $\mathrm{Hz}$ for alpha $(i=a),[13,30] \mathrm{Hz}$ for beta $(i=b)$ and $[30,40] \mathrm{Hz}$ for gamma $(i=g)$ bands, respectively i.e., $R I R_{d}$ represents RIR of delta band and so on.

\section{3) Maximum-to-Mean Power Ratio (mmrPS)}

The $m m r P S$ is ratio of the maximum power in a band to the mean of the total power. The mmrPS measures the relative strength and stretch in an EEG band. Thus, the $m m r P S$ can be expressed as

$$
\operatorname{mmrPS}_{i}=\max _{f_{i 1} \leq f \leq f_{i 2}}\left\{P_{x}(f) / \operatorname{mean}\left[P_{x}(f)\right]\right\}
$$

where $\left[f_{i 1}, f_{i 2}\right]$ are the edges of bands considered. In this study all the five bands, as in the case of RIR, are considered.

4) Peak BiSpectrum ( $p B S)$

The $p B S$ is the maximal bispectral value. Given a discretetime signal, $x(n), n=0,1,2, \ldots, N-1$, its bispectrum, $B_{x}\left(f_{1}, f_{2}\right)$ is estimated using the conventional (non-parametric) direct method [30], [31] and the $p B S$ is defined as

$$
p B S=\max _{f_{i 1} \leq f_{1}, f_{2} \leq f_{i 2}}\left\{B_{x}\left(f_{1}, f_{2}\right)\right\}
$$

where $\left[f_{i 1}, f_{i 2}\right]$ are the edges of bands considered. The maximal bispectral values in the delta-theta $(1-8 \mathrm{~Hz})$ band and in the alpha $(8-13 \mathrm{~Hz})$ band, namely the Slow Peak Bispectrum ( $p B S s l w)$, and the Fast Peak Bispectrum ( $p B S f s t)$ are considered in this study.

5) Peak BiCoherence $(p B C)$

The bicoherence, $b_{x}\left(f_{1}, f_{2}\right)$ is estimated by normalizing the bispectrum, $B_{x}\left(f_{1}, f_{2}\right)$ using the Kim-Powers [32] normalization factor and the $p B C$ can be expressed as

$$
p B C=\max _{f_{i 1} \leq f_{1}, f_{2} \leq f_{i 2}}\left\{b_{x}\left(f_{1}, f_{2}\right)\right\}
$$

where $\left[f_{i 1}, f_{i 2}\right]$ are the edges of bands considered. The maximal bicoherence values in the same bands as for the $p B S$ are considered. They are labeled as the Slow Peak BiCoherence $(p B C s l w)$, and the Fast Peak BiCoherence ( $p B C f s t$ ), respectively. Prior to selecting the peak values, the bicoherence values below the $95 \%$ statistically significant zero-bicoherence level [33] are zeroed out. 
6) Spectral Entropy (SE)

The $S E$ is a measure of spectral regularity and defined as [34]

$$
S E=-\left[1 / \ln \left(N_{f}\right)\right] \sum_{f} p_{x}(f) \ln \left[p_{x}(f)\right]
$$

where $p_{x}(f)$ is the probability density function in the frequency domain, estimated as

$$
p_{x}(f)=P_{x}(f) / \sum_{f} P_{x}(f)
$$

and $N_{f}$ is the number of frequency samples normalizing $S E$ to $[0,1]$. The $S E$ measures the complexity of the underlying processes that generate the signal. The larger is the value of $S E$, the more irregular is the spectrum of the signal and hence more complex is the generating system and vice versa.

\section{Extraction of features}

To prevent the effect of scaling on the values of the extracted features [35], each EEG record was first normalized.

All the features described above require the EEG signal to be stationary [36]. Since the EEG signals are non-stationary, they are often analyzed in segments in order to ensure the stationarity using a criterion of weak stationarity, which requires that the statistical parameters up to certain order remain (practically) constant over the entire period of the segment [37]. The most popularly used weak stationarity is the second-order stationarity which requires the secondorder statistics, mean and standard deviation, to remain constant at some prescribed significant level (e.g., $5 \%$ ) with the autocorrelation depending only on the time difference [38]. The test for detecting the stationary segment length was carried out as described in [37]. The dependency of the autocorrelation of each segment on time difference alone was checked as described in [35]. Segments of lengths $2 \mathrm{~s}$ and $4 \mathrm{~s}$ were chosen as stationary segments for this study. The 16 features described above were then extracted from every $2 \mathrm{~s}$ and $4 \mathrm{~s}$ segments of each channel record of $10 \mathrm{~min}$ duration. The value of each feature for each channel was estimated by averaging over all these segments and that for each subject, by averaging over all channels.

\section{E. Statistical evaluation of features}

The ability of the qEEG features (i.e., $P R I, R I R_{a}$, etc.) in discriminating the brain tumor case from the normal one is statistically assessed using the method of hypothesis-testing. A hypothetical test is a statistical test against a stated null hypothesis. A statistical hypothesis is an assumption or a guess made about a particular parameter of a population in a decision-making process [39]. The hypothetical test performed in this study may be formulated as follows [40]:

i. Identifying the parameter of interest for the test: The mean of a qEEG feature extracted from the scalp EEG signals of a set of 102 controlled subjects and a set of 100 brain tumor patients is the parameter of interest. ii. Stating the hypotheses for the test: If $\mu_{C i}$ and $\mu_{B i}$ are the means of a qEEG feature, $i$ estimated from a set of $n_{C}$ (102, in our study) controlled subjects and a set of $n_{B} \quad(100$, in our study) brain tumor patients, respectively, the null hypothesis, $H_{0}$ is that the means are equal i.e., the observed difference between the means is only by chance and the alternate hypothesis, $H_{1}$ is that the means are not equal i.e.,

$$
\begin{gathered}
H_{0}: \mu_{C i}=\mu_{B i} \text { or } \mu_{C i}-\mu_{B i}=0 \\
H_{1}: \mu_{C i} \neq \mu_{B i} \text { or } \mu_{C i}-\mu_{B i} \neq 0 \text { (two-tailed) }
\end{gathered}
$$

iii. Deciding on the significance level of the test: A desired level of significance, $\alpha$, which is the maximum probability of taking the risk to reject the null hypothesis when it is true [39], is chosen in the interval $(0,1)$. In this study, $\alpha=0.05$ i.e., a significance level of $5 \%$ is selected.

iv. Computing the test statistic: The following test statistic, $z_{0}$, often called the z-score, which is used to assess the strength of the evidence against $H_{0}$, is computed as in (10).

$$
z_{0}=\frac{\mu_{C i}-\mu_{B i}}{S E}
$$

where, in (10), $S E=\sqrt{\left(\sigma_{C i}^{2} / n_{C}\right)+\left(\sigma_{B i}{ }^{2} / n_{B}\right)}$ is the standard error with $\sigma_{C i}$ and $\sigma_{B i}$ being the observed standard deviations of the qEEG feature, $i$.

v. Computing the $p$-value: The $p$-value, which is the actual probability of taking the risk to reject the null hypothesis when it is true or, in other words, the observed level of significance [41], is computed as in (11).

$$
p=2\left[1-\Phi\left(z_{0}\right)\right]
$$

where, in (11), $\Phi\left(z_{0}\right)=P\left(Z \leq z_{0}\right)$ is the area under the standard normal curve below $Z=z_{0}$.

vi. Rejecting or accepting the null hypothesis: If $p<\alpha$, then the null hypothesis is rejected at $100(1-\alpha) \%$ confidence (or $100 \alpha \%$ significance) level.

Even the lower $p$-values do not remove the ambiguity whether the null hypothesis is false or the alternate hypothesis is true. Hence, following the suggestion from [42], the $95 \%$ confidence interval on the observed mean difference is also presented for a better understanding of the results of the hypothetical test carried out. The $95 \%$ confidence interval, $\mu_{i}$ on an observed mean difference, $\mu_{C i}-\mu_{B i}$ for a feature, $i$ is computed as in (12). This implies that there is a $95 \%$ chance that this interval would contain the mean difference, had the entire population been considered [42].

$$
\left(\mu_{C i}-\mu_{B i}\right)-z_{\alpha / 2} S E \leq \mu_{i} \leq\left(\mu_{C i}-\mu_{B i}\right)+z_{\alpha / 2} S E
$$




\section{RESULTS AND DISCUSSION}

Since the sample size in both cases is large enough $(>40)$ for the assumption of normality [39] of the estimated feature values, the statistical test is carried out with the two-tailed ztest at $95 \%$ confidence level.

Table 1. and Table 2. present the results of the statistical analysis of the candidate features from the frequencydomain for the $2 \mathrm{~s}$ and $4 \mathrm{~s}$ segmented analyses. From the fourth and fifth columns of these tables, it can be easily seen that there is not much difference between the $2 \mathrm{~s}$ and $4 \mathrm{~s}$ segmented analyses. Hence the $4 \mathrm{~s}$ segmented analysis is preferred due to the following facts: (i) this reduces the computational burden by reducing the number of segments to be analyzed, and (ii) this provides larger number of data points for a better estimation of the bispectral features. The latter point is evident from the statistical evaluation of bicoherence features, $p B C s l w$ and $p B C f s t$.

Comparing the results of statistical analysis presented in Table 1. and Table 2., it is inferred that the spectral features such as the $R I R$ in the delta band $\left(R I R_{d}\right)$ and the $m m r P S$ in the delta band $\left(m m r P S_{d}\right)$ show the dominance of the slow activities in the brain tumor. The features extracted from the bispectrum, namely $p B S s l w$ and $p B S f s t$ are statistically significant while those from the bicoherence i.e., the normalized bispectrum, namely $p B S s l w$ and $p B C f s t$ are not in Table 1. However, the increase in the data length from $2 \mathrm{~s}$ to $4 \mathrm{~s}$ tremendously increases the significance of these features, which is clearly evident from Table 2 .

According to the results of statistical analysis presented in Table 1. and Table 2., the feature, PRI does not show statistically significant difference between the brain tumor case and the normal one $(p>0.05)$. But the loss in the high frequency power (e.g., $R I R_{a} \& m m r P S_{a}$ in alpha band) is more discriminating $(p<0.0001)$ than the gain in the low frequency power (e.g., $R I R_{d} \& m m r P S_{d}$ in delta band) $(p<0.001)$. The feature, $P R I$, though $p>0.05$, may also be considered significant due to a large difference in the mean values. Fig.1. and Fig.2. show the plots of calculated feature values from two-second and four-second segments, respectively.

Table 1. Results of statistical analysis of features from two-second segments.

\begin{tabular}{ccccc}
\hline \multirow{2}{*}{ Feature } & Normal & Brain Tumor & p-value & CI $^{\mathrm{c}}$ \\
\cline { 2 - 3 } & Mean (SD) & Mean (SD) & & \\
\hline$P R I$ & $0.7946(0.4664)$ & $2.8505(10.5275)$ & 0.0511 & $(-0.0095,4.1212)$ \\
$R I R_{d}$ & $0.1847(0.1242)$ & $0.2748(0.2125)$ & $0.0002^{\mathrm{b}}$ & $(0.0420,0.1382)$ \\
$R I R_{t}$ & $0.1469(0.0317)$ & $0.1625(0.0747)$ & 0.0537 & $(-0.0002,0.0315)$ \\
$R I R_{a}$ & $0.3987(0.1383)$ & $0.3044(0.1506)$ & $0.0000^{\mathrm{b}}$ & $(0.0544,0.1342)$ \\
$R I R_{b}$ & $0.0854(0.0146)$ & $0.0741(0.0579)$ & 0.0581 & $(-0.0004,0.0230)$ \\
$R I R_{g}$ & $0.0023(0.0035)$ & $0.0020(0.0025)$ & 0.4461 & $(-0.0005,0.0012)$ \\
$m m r P S_{d}$ & $3.7076(2.4869)$ & $5.6333(4.8675)$ & $0.0004^{\mathrm{b}}$ & $(0.8566,2.9949)$ \\
$m m r P S_{t}$ & $3.3311(0.5646)$ & $3.6861(1.6128)$ & $0.0375^{\mathrm{a}}$ & $(0.0205,0.6896)$ \\
$m m r P S_{a}$ & $6.9914(2.3435)$ & $5.3728(2.3942)$ & $0.0000^{\mathrm{b}}$ & $(0.9651,2.2721)$ \\
$m m r P S_{b}$ & $0.9283(0.2942)$ & $0.7916(0.5440)$ & $0.0268^{\mathrm{a}}$ & $(0.0157,0.2576)$ \\
$m m r P S_{g}$ & $0.0293(0.0410)$ & $0.0255(0.0316)$ & 0.4630 & $(-0.0063,0.0139)$ \\
$p B S s l w$ & $5778.3231(1268.4573)$ & $6715.4363(2330.4213)$ & $0.0004^{\mathrm{b}}$ & $(418.2482,1455.9782)$ \\
$p B S f s t$ & $2468.8109(866.6290)$ & $1950.9366(1114.7279)$ & $0.0002^{\mathrm{b}}$ & $(242.1570,793.5917)$ \\
$p B C s l w$ & $0.5228(0.0981)$ & $0.5404(0.0473)$ & 0.1034 & $(-0.0036,0.0388)$ \\
$p B C f s t$ & $0.1886(0.2530)$ & $0.1346(0.1149)$ & 0.0500 & $(0.0000,0.1080)$ \\
$S E$ & $0.5924(0.0272)$ & $0.5904(0.0441)$ & 0.7037 & $(-0.0082,0.0121)$ \\
\hline SD Standard Deviation; ${ }^{\mathrm{a}} \mathrm{p}<0.05 ;{ }^{\mathrm{b}} \mathrm{p}<0.01 ;{ }^{\mathrm{c}} 95 \%$ Confidence Interval on the mean difference
\end{tabular}

Table 2. Results of statistical analysis of features from four-second segments

\begin{tabular}{|c|c|c|c|c|}
\hline \multirow{2}{*}{ Feature } & Normal & Brain Tumor & \multirow{2}{*}{ p-value } & \multirow{2}{*}{$\mathbf{C I}^{\mathrm{c}}$} \\
\hline & Mean (SD) & Mean & & \\
\hline PRI & $0.7407(0.4435)$ & $2.8856(11.0170)$ & 0.0517 & $(-0.0161,4.3059)$ \\
\hline$R I R_{d}$ & $0.1860(0.1268)$ & $0.2811(0.2210)$ & $0.0002^{\mathrm{b}}$ & $(0.0452,0.1449)$ \\
\hline$R I R_{t}$ & $0.1397(0.0337)$ & $0.1553(0.0777)$ & 0.0649 & $(-0.0010,0.0322)$ \\
\hline$R I R_{a}$ & $0.3967(0.1454)$ & $0.2967(0.1550)$ & $0.0000^{\mathrm{b}}$ & $(0.0585,0.1415)$ \\
\hline$R I R_{b}$ & $0.0791(0.0133)$ & $0.0686(0.0544)$ & 0.0585 & $(-0.0004,0.0216)$ \\
\hline$R I R_{g}$ & $0.0021(0.0033)$ & $0.0018(0.0024)$ & 0.4374 & $(-0.0005,0.0011)$ \\
\hline$m m r P S_{d}$ & $4.7866(3.2433)$ & $6.5928(6.9927)$ & $0.0189^{\mathrm{a}}$ & $(0.2981,3.3144)$ \\
\hline$m m r P S_{t}$ & $3.7347(0.7521)$ & $4.1731(2.1314)$ & 0.0522 & $(-0.0042,0.8808)$ \\
\hline$m m r P S_{a}$ & $9.4331(3.8521)$ & $6.8017(3.5078)$ & $0.0000^{\mathrm{b}}$ & $(1.6157,3.6470)$ \\
\hline$m m r P S_{b}$ & $1.0002(0.3005)$ & $0.8709(0.6049)$ & 0.0551 & $(-0.0028,0.2614)$ \\
\hline$m m r P S_{g}$ & $0.0329(0.0485)$ & $0.0275(0.0343)$ & 0.3607 & $(-0.0062,0.0170)$ \\
\hline$p B S s l w$ & 4889.9794 (1162.6352) & $5542.0564(2549.2081)$ & $0.0197^{\mathrm{a}}$ & $(103.8587,1200.2954)$ \\
\hline$p B S f s t$ & $2181.6919(1042.7602)$ & $1626.4340(1002.7101)$ & $0.0001^{\mathrm{b}}$ & $(273.1690,837.3467)$ \\
\hline$p B C s l w$ & $0.3652(0.0812)$ & $0.3939(0.0519)$ & $0.0027^{\mathrm{b}}$ & $(0.0099,0.0474)$ \\
\hline$p B C f s t$ & $0.1427(0.2143)$ & $0.0957(0.0942)$ & $0.0430^{\mathrm{a}}$ & $(0.0015,0.0925)$ \\
\hline$S E$ & $0.5722(0.0302)$ & $0.5705(0.0486)$ & 0.7608 & $(-0.0094,0.0129)$ \\
\hline
\end{tabular}

SD Standard Deviation; ${ }^{\mathrm{a}} \mathrm{p}<0.05 ;{ }^{\mathrm{b}} \mathrm{p}<0.01 ;{ }^{\mathrm{c}} 95 \%$ Confidence Interval on the mean difference 

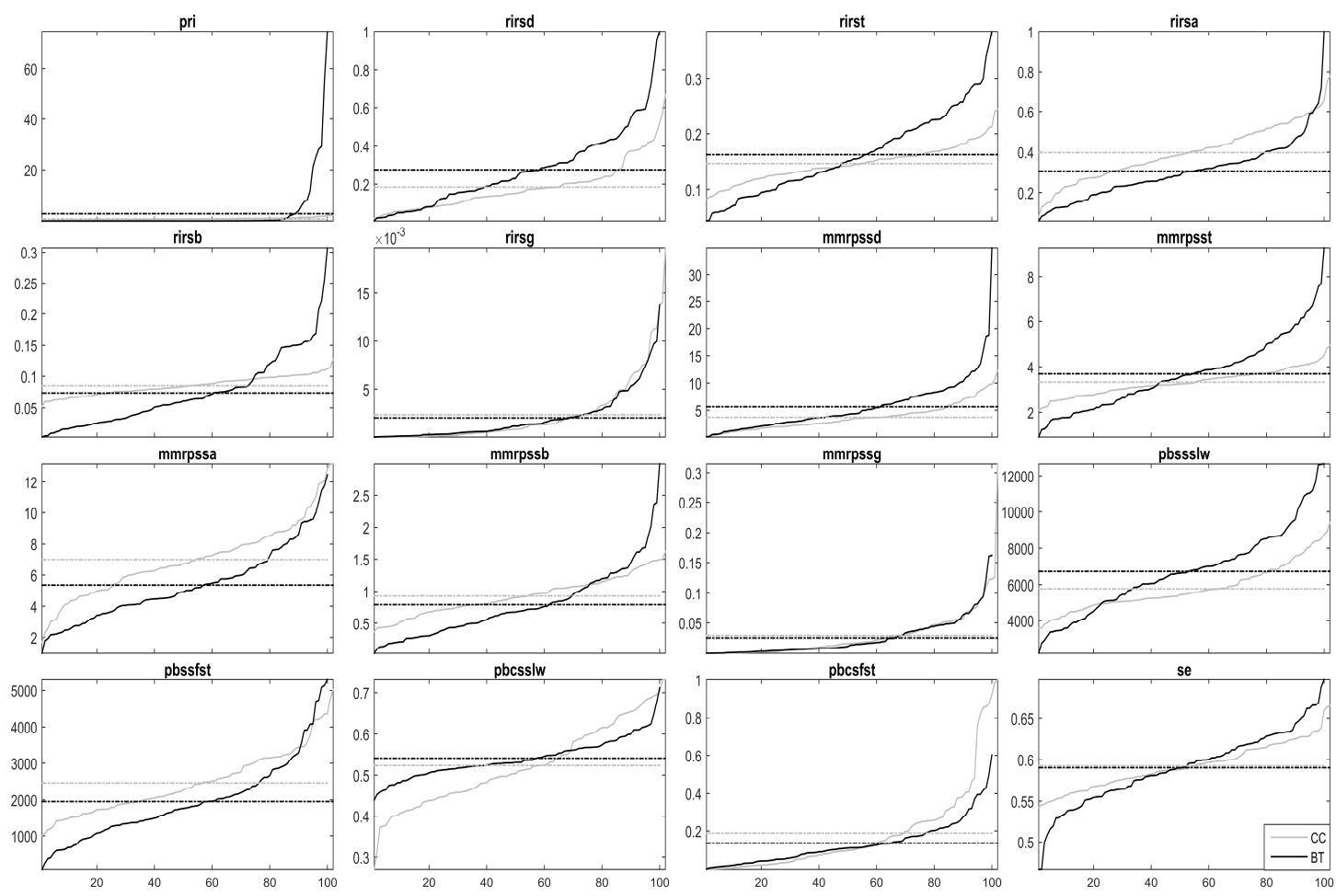

Fig.1. Plots of the calculated feature values from two-second segments: (i) the horizontal axis shows the sample number, (ii) the vertical number shows the feature values, (iii) the darker line corresponds to the feature values of brain tumor (BT) case, (iv) the lighter line corresponds to the feature values of controlled case (CC) and (v) the horizontal broken lines indicate the means of respective cases.
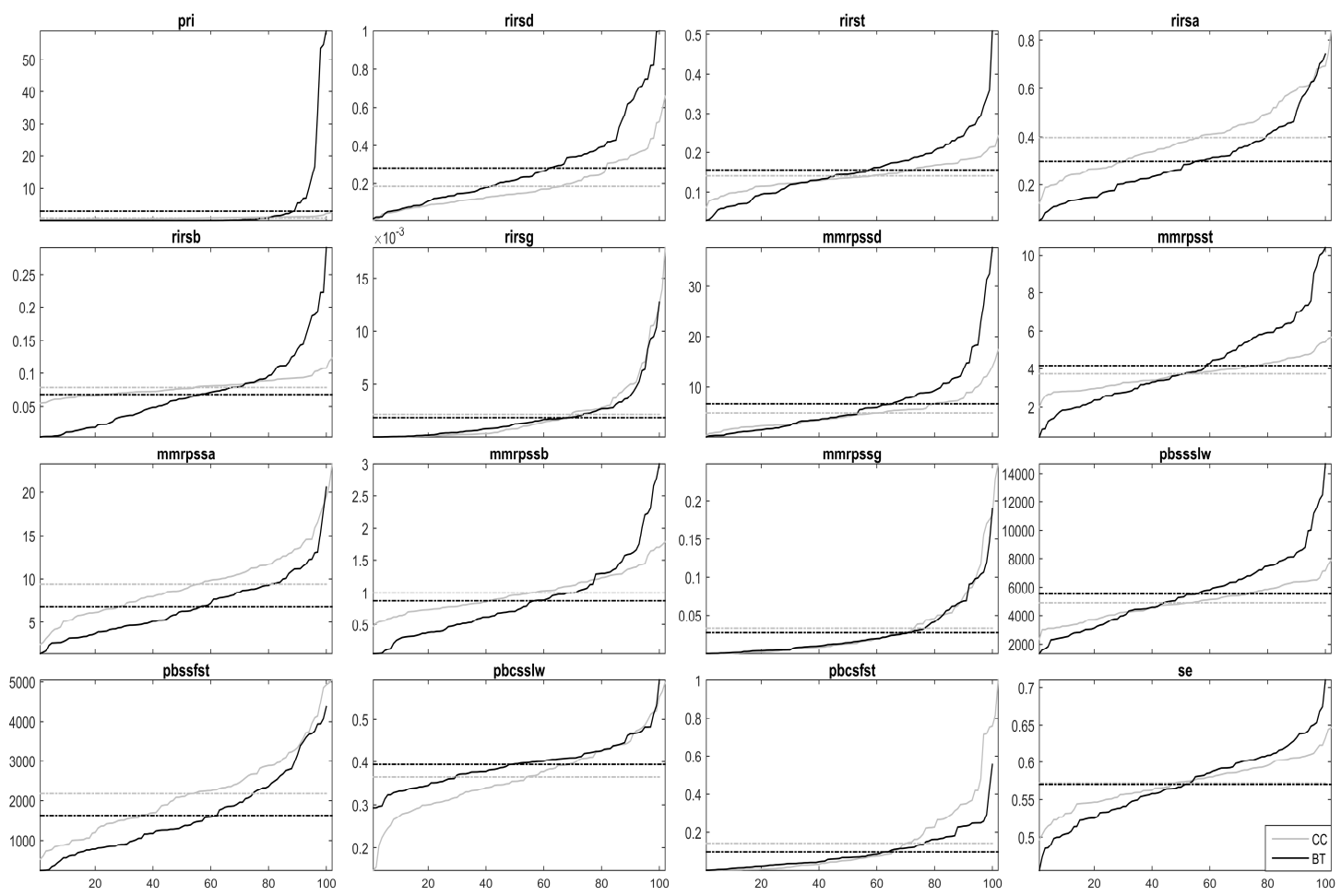

Fig.2. Plots of the calculated feature values from four-second segments. 


\section{CONCLUSIONS}

Several candidate features from the frequency-domain were investigated and the hypothetical test on the difference in the means of these features in discriminating a brain tumor patient from a normal subject was performed. The results, on the average, encourage the use of scalp EEG for the diagnosis of brain tumor. The statistically significant sample size (100 brain tumor and 102 normal cases) ensures the reliability of the results obtained.

Since a set of features which are more relevant to the classes but less redundant to each other is required for a successful classification and the mere statistical inference on the mean difference cannot assess this, a well-defined feature selection process would be an appropriate approach to identify a subset containing the most discriminating features. This, along with an appropriate discriminant analysis, would prove the usability of these features for the clinical purpose.

\section{ACKNOWLEDGMENT}

The authors sincerely thank the Ethical Committee members of Madras Medical College (MMC), Chennai, India, for having granted permission to collect the data required for this research study from $\mathrm{MMC}$.

\section{REFERENCES}

[1] Dobrovoljac, M., Hengartner, H., Boltshauser, E., Grotzer, M.A. (2002). Delay in the diagnosis of paediatric brain tumours. European Journal of Pediatrics, 161 (12), 663-667.

[2] Edgeworth, J., Bullock, P., Bailey, A., Gallagher, A., Crouchman, M. (1996). Why are brain tumours still being missed? Archives of Disease in Childhood, 74, 148-151.

[3] Flores, L.E., Williams, D.L., Bell, B.A., O'Brien, M., Ragab, A.H. (1986). Delay in the diagnosis of pediatric brain tumors. American Journal of Diseases of Children, 140 (7), 684-686.

[4] Reulecke, B.C., Erker, C.G., Fiedler, B.J., Niederstadt, T.U., Kurlemann, G. (2008). Brain tumors in children: Initial symptoms and their influence on the time span between symptom onset and diagnosis. Journal of Child Neurology, 23 (2), 178-183.

[5] Musella, A. (2010-2013). Brain Tumor Symptoms Survey Results. http://www.virtualtrials.com/ braintumorsymptomssurvey.cfm.

[6] Husing, B., Jancke, L., Tag, B. (2006). Impact Assessment of Neuroimaging. Final Report. vdf Hochschulverlag AG an der ETH Zürich. http://www.vdf.ethz.ch/service/3065/3065_Neuroimag ing_OA.pdf.

[7] Walter, G. (1936). The location of cerebral tumors by electroencephalography. Lancet, 228 (5893), 305-308.

[8] Accolla, E.A., Kaplan, P.W., Maeder-Ingvar, M., Jukopila, S., Rossetti, A.O. (2011). Clinical correlates of frontal intermittent rhythmic delta activity (FIRDA). Clinical Neurophysiology, 122, 27-31.
[9] Bagchi, B.K., Kooh, K.A., Selving, B.T., Calhoun, H.D. (1961). Subtentorial tumors and other lesions: An electroencephalographic study of 121 cases. Electroencephalography and Clinical Neurophysiology, 13 (2), 180-192.

[10] Silverman, D., Sannit, T., Ainspac, S., Freedman, S. (1960). Serial electroencephalography in brain tumors and cerebrovascular accidents. Archives of Neurology, 2 (2), 122-129.

[11] Fischer-Williams, M. (2004). Brain tumors and other space-occupying lesions. In Electroencephalography: Basic Principles, Clinical Applications, and Related Fields. Lippincott Williams \& Wilkins, 305-321.

[12] Kumar, S. (2005). Asymmetric depression of amplitude in EEG leading to a diagnosis of ipsilateral cerebral tumor. Annals of Indian Academy of Neurology, 8, 33-36.

[13] O'Connor, S.C. Robinson, P.A. (2005). Analysis of the electroencephalographic activity associated with thalamic tumors. Journal of Theoretical Biology, 233, 271-286.

[14] Watemberg, N., Alehan, F., Dabby, R., Lerman-Sagie, T., Pavot, P., Towne, A. (2002). Clinical and radiologic correlates of frontal intermittent rhythmic delta activity. Journal of Clinical Neurophysiology, 19 (6), 535-539.

[15] Chetty, S., Venayagamoorthy, G.K. (2002). A neural network based detection of brain tumours using electroencephalography. In Proceedings of IASTED International Conference Artificial Intelligence and Soft Computing, 17-19 July 2002, Banff, Canada, 391396.

[16] Habl, M., Bauer, Ch., Ziegaus, Ch., Lang, E.W., Schulmeyer, F. (200). Can ICA help identify brain tumor related EEG signals? In Proceedings of Second International Workshop on Independent Component Analysis and Blind Signal Separation, 19-22 June 2000, Helsinki, Finland, 609-614.

[17] Karameh, F.N., Dahleh, M.A. (2000). Automated classification of EEG signals in brain tumor diagnostics. In Proceedings of the 2000 American Control Conference, 28-30 June 2000, Chicago, Illinois, 4169-4173.

[18] Murugesan, M., Sukanesh, R. (2009). Automated detection of brain tumor in EEG signals using artificial neural networks. In Proceedings of 2009 International Conference on Advances in Computing, Control, and Telecommunication Technologies, 28-29 December 2009, Trivandrum, Kerala. IEEE, 284-288.

[19] Nagata, K., Gross, C.E., Kindt, G.W., Geier, M.J., Adey, G.R. (1985). Topographic electroencephalographic study with power ratio index mapping in patients with malignant brain tumors. Neurosurgery, 17 (4), 613-619.

[20] Silipo, R., Deco, G., Bartsch, H. (1999). Brain tumor classification based on EEG hidden dynamics. Intelligent Data Analysis, 3 (4), 287-306.

[21] Ko, D.Y. (2009). EEG in Brain Tumors. http://emedicine.medscape.com/article/1137982overview. 
[22] Picot, A., Charbonnier, S., Caplier. A. (2009). Monitoring drowsiness on-line using a single encephalographic channel. In Biomedical Engineering. In-Tech, 145-164. http://cdn.intechopen.com/pdfswm/8799.pdf.

[23] Nunez, P.L., Srinivasan, R. (2006). Electric Fields of the Brain: The Neurophysics of EEG, (2nd Ed.). Oxford University Press.

[24] Lofhede, J. (2007). Classification of Burst and Suppression in the Neonatal EEG. Unpublished doctoral dissertation, Chalmers University of Technology, Goteborg, Sweden.

[25] Salido-Ruiz, R., Ranta, R., Louis-Dorr, V. (2011). EEG montage analysis in the blind source separation framework. Biomedical Signal Processing and Control, 6 (1), 77-84.

[26] Hirsch, L., Brenner, R. (2009). Atlas of EEG in Critical Care, (1st Ed.). John Wiley \& Sons.

[27] Gudmundsson, S., Runarsson, T.P., Sigurdsson, S., Eiriksdottir, G., Johnsen, K. (2007). Reliability of quantitative EEG features. Clinical Neurophysiology, 118, 2162-2171.

[28] Proakis, J.G., Manolakis, D.G. (2000). Digital Signal Processing Principles, Algorithms, and Applications, (3rd Ed.). Prentice-Hall of India.

[29] Bao, F.S., Gao, J.M., Hu, J., Lie, D.Y.C., Zhang, Y., Oommen, K.J. (2009). Automated epilepsy diagnosis using interictal scalp EEG. In Proceedings of 31 st Annual International Conference of the IEEE EMBS, 2-6 September 2009, Minneapolis, Minnesota. IEEE, 6603-6607.

[30] Nikias, C.L., Raghuveer, M.R. (1987). Bispectrum estimation: A digital signal processing framework. Proceedings of the IEEE, 75 (7), 869-891.

[31] Petropulu, A.P. (2000). Higher-order spectral analysis. In The Biomedical Engineering Handbook. CRC Press, 1-17.

[32] Kim, Y.C., Powers, E.J. (1979). Digital bispectral analysis and its applications to nonlinear wave interactions. IEEE Transactions on Plasma Science, 7 (2), 120-131.
[33] Elgar, S., Guza, R.T. (1988). Statistics of bicoherence. IEEE Transactions on Acoustics, Speech, and Signal Processing, 36 (10), 1667-1668.

[34] Mahon, P., Greene, B.R., Greene, C., Boylan, G.B. Shorten, G.D. (2008). Behaviour of spectral entropy, spectral edge frequency $90 \%$, and alpha and beta power parameters during low-dose propofol infusion. British Journal of Anaesthesia, 101 (2), 213-21.

[35] Breakspear, M., Terry, J.R. (2002). Detection and description of non-linear interdependence in normal multichannel human EEG data. Clinical Neurophysiology, 113, 735-753.

[36] Pereda, E., Cruz, D.M., Manas, S., Garrido, J.M., Lopez, S., Gonzalez, J.J. (2006). Topography of EEG complexity in human neonates: Effect of the postmenstrual age and the sleep state. Neuroscience Letters, 394, 152-157.

[37] Blanco, S., Garcia, H., Quiroga, R.Q., Romanelli, L., Rosso, O.A. (1995). Stationaity of the EEG series. IEEE Engineering in Medicine and Biology, 14 (4), 395-399.

[38] Jeong, J., Gore, J.C., Peterson, B.S. (2002). A method for determinism in short time series, and its application to stationary EEG. IEEE Transactions on Biomedical Engineering, 49 (11), 1374-79.

[39] Spiegel, M.R., Schiller, J., Srinivasan, R.A. (2001). Probability and Statistics, (Schaum). New Delhi, India: McGraw Hill.

[40] Montgomery, D.C., Runger, G.C. (2003). Applied Statistics and Probability for Engineers, (3rd Ed.). John Wiley \& Sons.

[41] DeGroot, M.H., Schervish, M.J. (2012). Probability and Statistics (4th Ed.). Addison-Wesley.

[42] Gardner, M.J., Altman, D.G. (2005). Confidence intervals rather than $\mathrm{P}$ values. In Statistics with Confidence. Bristol: BMJ, 15-27.

Received January 09, 2015. Accepted August 12, 2015. 